\title{
AN UNSOLVABLE EQUATION ${ }^{1}$
}

\section{P. R. GARABEDIAN}

Abstract. A linear partial differential equation that is not solvable is obtained by a very simple transformation of the CauchyRiemann equations.

It seems worth while to call attention to the following simple example, which is implicit in the work of Lewy [1] and Nirenberg and Treves [2]:

THEOREM. The linear partial differential equation

$$
u_{x}+i x u_{y}=|x y|
$$

is not classically solvable in any neighborhood of the origin.

Proof. Since the odd part with respect to $x$ of any solution is also a solution, the expression

$$
U(x, y)=u(x, y)-u(-x, y)+i y|y|
$$

satisfies the homogeneous equation

$$
U_{x}+i x U_{y}=0
$$

for $x>0$. Therefore it is an analytic function of the complex variable $z=x^{2} / 2+i y$ in some neighborhood of the origin within the right halfplane, and moreover its real part vanishes on the imaginary axis. By the Schwarz principle of reflection $U$ can be continued analytically into the left half-plane, so $U(0, y)$ must be an analytic function of $y$ for small $|y|$. But this contradicts the fact that the second derivative of $y|y|$ is discontinuous at the origin.

Smoother inhomogeneous terms such as $|x y|^{3}$ or $\exp \left(-1 / y^{2}\right)$ provide unsolvable equations that are almost as easy to analyze. More specifically, in the harder case of $\exp \left(-1 / y^{2}\right)$ one has only to consider the analytic function

$$
U\left(\frac{x^{2}}{2}+i y\right)=\frac{1}{\pi i} \int_{-x}^{x} \frac{u(\xi, y) \xi d \xi}{\left(x^{2}-\xi^{2}\right)^{1 / 2}}+\int \exp \left(-1 / y^{2}\right) d y .
$$

Received by the editors October 28, 1969.

A MS Subject Classifications. Primary 3501, 3530; Secondary 3028.

Key Words and Phrases. Unsolvable equation, odd part, complex variable, Schwarz principle of reflection.

1 This research was supported by the U.S. Atomic Energy Commission under contract AT (30-1)-1480-V with New York University. 
Also, without looking at such a complicated integral it is easy to see that the infinitely differentiable equation

$$
u_{x}+i\left(x e^{-1 / x^{2}} /|x|\right) u_{y}=\exp \left(-1 / x^{2}-1 / y^{2}\right)
$$

is not solvable at the origin.

\section{REFERENCES}

1. H. Lewy, An example of a smooth linear partial differential equation without solution, Ann. of Math. (2) 66 (1957), 155-158. MR 19, 551.

2. L. Nirenberg and F. Treves, Solvability of a first order linear partial differential equation, Comm. Pure Appl. Math, 16 (1963), 331-351. MR 29 \#348.

Courant Institute of Mathematical Sciences, New York University, NEW YoRK, NEW YORK 10012 